\title{
Efeito do Ramipril e da Sinvastatina sobre o Estresse Oxidativo de Ratos Diabéticos
}

\begin{abstract}
RESUMO
Objetivo: Avaliar se o ramipril, isoladamente ou em combinação com a sinvastatina, seria capaz de reduzir o estresse oxidativo de ratos diabéticos pela estreptozotocina (STZ). Métodos: As drogas foram administradas a ratos diabéticos por duas semanas; o estresse oxidativo foi medido por dosagem de capacidade antioxidante total plasmática (TRAP) e malonaldeído (MDA). Resultados: $O$ ramipril usado isoladamente foi capaz de aumentar significativamente as defesas antioxidantes do rato diabético; a sinvastatina isoladamente ou combinada ao ramipril em tomadas separadas não produziu efeito significativo sobre o estresse oxidativo; a administração simultânea de ramipril e sinvastatina reduziu as defesas antioxidantes plasmáticas de ratos com diabetes melito químico. Conclusões: Os dados do presente estudo corroboram o efeito positivo do ramipril sobre a defesa antioxidante do plasma, mas não confirmam um possível efeito benéfico da sinvastatina no modelo. Pesquisas adicionais são necessárias para clarificar a paradoxal redução da TRAP verificada pela administração simultânea das drogas. (Arq Bras Endocrinol Metab 2008; 52/7:1131-1138)
\end{abstract}

Descritores: Ramipril; Sinvastatina; Diabetes melito; Antioxidantes

\begin{abstract}
Effects of Ramipril and Simvastatin on the Oxidative Stress of Diabetic Rats. Objective: To evaluate if ramipril, with or without simultaneous use of simvastatin, would be capable of reducing oxidative stress of streptozotocin (STZ) induced diabetic rats. Methods: The drugs were given to the diabetic rats for 2 weeks; oxidative stress was measured by dosage of total plasma antioxidant capacity (TRAP) and malondialdehyde (MDA). Results: Ramipril, used alone, was capable of significantly increasing the antioxidative defenses of the diabetic rat; simvastatin, given alone or combined with ramipril in separate administrations, did not produce any significant effect on the oxidative stress; concomitant administration of ramipril and simvastatin significantly reduced the antioxidative plasmatic defenses of rats with chemically induced diabetes mellitus Conclusions: Our data corroborate the positive effect of ramipril upon plasma antioxidative defenses but did not confirm a possible beneficial effect of simvastatin in the model. More research is needed to clarify the paradoxal TRAP reduction verified with simultaneous administration of the drugs. (Arq Bras Endocrinol Metab 2008; 52/7:1131-1138)
\end{abstract}

Keywords: Ramipril; Simvastatin; Diabetes mellitus; Antioxidants

\section{INTRODUÇÃO}

A lém da alta morbidade acarretada pelas complicações crônicas da doença, os pacientes diabéticos apresentam mortalidade muito elevada em relação à da população geral. A principal causa desta é o elevado número de eventos original

JosÉ A. Z. ELIAS

VINICIUS D. A. DELFINO

DÉCIO S. BARBOSA

BRUNO A. FABRIS

TIEMI Matsuo

Departamento de Farmácia da Universidade Norte do Paraná (Unopar), (JAZE); Departamento de Nefrologia da Universidade Estadual de Londrina (UEL), (VDAD); Departamento de Patologia, Análises Clínicas e Toxicológicas da UEL (DSB, BAF); Departamento de Estatística e Matemática Aplicada da UEL (TM); Londrina, PR, Brasil.

Recebido em 28/6/2007 Aceito em 19/8/2008 
cardiovasculares fatais, decorrentes de aterosclerose acelerada. Entre os vários fatores envolvidos nesta aterosclerose acelerada estão: hiperglicemia, hipercolesterolemia, hipertensão arterial, disfunção endotelial e aumento do estresse oxidativo (1-4).

Os anti-hipertensivos inibidores da enzima de conversão (IECA)/bloqueadores dos receptores $\mathrm{AT}_{1}$ da angiotensina II $\left(\right.$ BRAT $\left._{1}\right)$ possuem outras ações em adição ao efeito redutor da pressão arterial, incluindo-se atividade antioxidante, melhora da disfunção endotelial, atenuação da progressão da aterosclerose, nefro e cardioproteções (5-7). Também para as estatinas, drogas que reduzem a formação de colesterol pelo bloqueio da 3-hidroxi-3-metilglutaril CoA redutase (HMG-CoA redutase), são descritas propriedades farmacológicas adicionais, como vasodilatação, ação antitrombótica, antiinflamatória, antiproliferativa e ação redutora sobre o estresse oxidativo $(8,9)$.

A hipertensão e a dislipidemia são freqüentemente observadas em diabéticos, sendo os inibidores de enzima de conversão e as estatinas amplamente utilizados nesta população, promovendo redução nas complicações crônicas e na mortalidade destes pacientes (10-12).

Por causa da íntima correlação entre sistema reninaangiotensina-aldosterona (SRAA), lipídeos séricos e estresse oxidativo, o bloqueio simultâneo do SRAA e da síntese de colesterol pela HMG-CoA redutase esperado é promover efeitos antioxidantes sinérgicos (10-14).

Foi realizado estudo experimental com o objetivo de avaliar se a adição de um inibidor da enzima de conversão - o ramipril - à uma estatina - a sinvastatina - seria capaz de promover maior redução no estresse oxidativo do rato diabético pela estreptozotocina do que cada uma das drogas isoladamente, estresse oxidativo este medido pelos níveis plasmáticos de capacidade antioxidante total plasmática (TRAP) e malonaldeído (MDA).

\section{MÉTODOS}

\section{Animais utilizados}

Foram utilizados em todos os experimentos ratos Lewis (LEW), isogênicos, machos, com idade entre 36 e 42 semanas, alimentados com ração apropriada para ratos e água ad libitum. O manuseio dos animais foi realizado em concordância com os princípios éticos para manipulação de animais de experimentação (15).

\section{Drogas utilizadas}

A estreptozotocina foi obtida da Sigma (Estados Unidos) e a heparina não-fracionada da Cristália (Brasil). O éter etílico para anestesia inalatória foi adquirido com a Biotech (Paraná, Brasil). Durante todo o estudo, uma única apresentação comercial de sinvastatina (Sinvalip ${ }^{\circledR}$, comprimidos de $40 \mathrm{mg}$ ) e de ramipril (Naprix ${ }^{\circledR}$, comprimidos de $5 \mathrm{mg}$ ) foram utilizadas. Os comprimidos foram triturados até o estado de pó, por meio de graal e pistilo, e depois foram incorporados em solução de CMC $0,5 \%$ e suas doses eram adequadas ao peso do animal.

\section{Estabelecimento do diabetes melito experimental}

O estabelecimento do diabetes melito experimental foi induzido nos animais pela administração intraperitoneal de $50 \mathrm{mg} / \mathrm{kg}$ de estreptozotocina (STZ). Foram considerados diabéticos os ratos que após duas semanas da administração de STZ apresentaram perda de peso corporal e níveis de glicemia, obtida pela seção feita sob anestesia inalatória com éter etílico de pequeno fragmento distal de suas caudas, maior ou igual a $300 \mathrm{mg} / \mathrm{dL}$, em duas determinações consecutivas com glicosímetro digital ACCU-CHEK.

\section{Coleta de sangue para determinação do estado oxidativo plasmático}

As amostras sangüíneas foram coletadas dos ratos sob anestesia inalatória com éter etílico, 5 a 8 minutos após injeção intraperitoneal de heparina sódica não-fracionada, diluída 1:10 com água destilada estéril, na dose de $500 \mathrm{UI} / \mathrm{kg}$. Os animais tiveram suas caudas seccionadas em seus terços médios, com lâmina de bisturi nova, e de cada animal foram coletados, em tubos com ácido etileno diamino tetracético (EDTA), $4 \mathrm{~mL}$ de sangue, cujo plasma foi imediatamente separado e congelado a $-20^{\circ} \mathrm{C}$, em tubo de ensaio previamente codificado, por período de até 30 dias, para posterior análise dos níveis de MDA e da TRAP.

\section{Determinação do estresse oxidativo plasmático}

O estresse oxidativo plasmático foi avaliado pela determinação plasmática dos níveis de MDA e pela estimativa da TRAP em ratos adultos (heparinizados e não-heparinizados) e em ratos diabéticos dos diferentes grupos de tratamento após duas semanas de administração das drogas ou do placebo ao final do 
experimento. As determinações dos níveis de MDA e TRAP foram feitas por um colaborador, sem o conhecimento da codificação dos tubos contendo as amostras plasmáticas.

\section{$M D A$}

Para a determinação da quantidade de MDA foram incubados a $90{ }^{\circ} \mathrm{C}$, por 45 minutos, $800 \mu \mathrm{L}$ de plasma de rato com $1 \mathrm{~mL}$ de ácido tiobarbitúrico, os tubos foram então resfriados e acrescentado $0,2 \mathrm{~mL}$ de $\mathrm{NaCl}$ saturado e 2,0 mL de N-butanol. Os tubos foram então agitados por 40 segundos e, logo depois, centrifugados a $3.000 \mathrm{rpm}$ por 10 minutos. A densidade óptica do sobrenadante foi lida por meio de espectrofotometria, nos comprimentos de onda de 535 e $572 \mathrm{~nm}$, contra o branco. O valor do resultado de MDA foi corrigido pela quantidade de proteína, determinada pelo método de Lowry, da amostra.

\section{TRAP}

Foi avaliado por quimiluminescência $(\mathrm{QL})$ pela técnica de Repetto modificada por Barbosa e cols. (16), no qual ao meio de reação contendo $1,8 \mathrm{~mL}$ de tampão glicina $(0,1 \mathrm{M}, \mathrm{pH} 8,6)$ foram acrescidos $100 \mu \mathrm{l}$ de luminol em solução aquosa $200 \mu \mathrm{M}, 20 \mu \mathrm{L}$ de plasma e $100 \mu \mathrm{L}$ de solução aquosa de 2,2' azo-bis (2-amidinopropano) 200 $\mathrm{mM}$. O sistema foi calibrado com análogo de vitamina $\mathrm{E}$ (trolox), $100 \mu \mathrm{L}$ na concentração de $20 \mu \mathrm{M}$ em tampão glicina $\mathrm{pH} 8$,6. Uma comparação do tempo de indução, depois da adição de concentrações conhecidas de trolox, e plasma permitiu obter valores de TRAP em equivalentes de trolox segundo a equação: TRAP $(\mu \mathrm{M}$ trolox $)=$ $\mathrm{D} \times\left(\mathrm{t}_{\text {amostra }} / \mathrm{t}_{\text {Trolox }}\right)$. Na equação, $\mathrm{D}$ é um fator de diluição da amostra no meio de reação; $t_{\text {amostra }}$ é o tempo de indução promovido pela adição da amostra de plasma; $\mathrm{t}_{\text {Trolox }}$ é o tempo induzido por $1 \mu \mathrm{M}$ de trolox. Os resultados foram expressos em $\mu \mathrm{M}$ de trolox. As determinações foram realizadas em um contador $\beta$ marca Beckman ${ }^{\circledR}$ (Estados Unidos) modelo LS 6000, em um modo de contagem não-coincidente por 25 minutos e faixa de resposta entre 300 a $620 \mathrm{~nm}$.

\section{Grupos de estudo}

Avaliação do efeito da injeção intraperitoneal de heparina não-fracionada sobre as defesas antioxidantes e o estresse oxidativo de ratos LEW isogênicos normais (sem diabetes químico)

Cinco animais receberam, intraperitonealmente (IP), heparina não-fracionada, diluída $1 / 10$ em água desti- lada estéril, na dose de $500 \mathrm{UI} / \mathrm{kg}$ de peso. Após 5 a 8 minutos da administração de heparina, sob anestesia inalatória com éter etílico, pelos vasos caudais, amostras sangüíneas para a determinação de TRAP e MDA foram obtidas dos animais. Cinco animais foram injetados com água destilada ip para funcionarem como controles. No entanto, um animal morreu durante a anestesia, restando quatro animais para a análise estatística.

\section{Estudo experimental propriamente dito}

Trinta e oito ratos diabéticos pela estreptozotocina foram utilizados. Os animais foram aleatoriamente alocados nos grupos de tratamento a seguir descritos: a) controles diabéticos $(\mathrm{CD})$; b) diabéticos tratados com ramipril (R); c) diabéticos tratados com sinvastatina (S); d) diabéticos tratados com ramipril e sinvastatina juntos na mesma administração (RSJ); e e) diabéticos tratados com ramipril e sinvastatina em administrações separadas (RSS). Os animais dos diferentes grupos receberam diariamente, por duas semanas, por gavagem, as drogas em estudos, diluídas em carboxi-metil-celulose (CMC) $0,5 \%$ (ramipril $3 \mathrm{mg} / \mathrm{kg} / \mathrm{dia}$ e/ou sinvastatina $2 \mathrm{mg} / \mathrm{kg} /$ dia), na dependência do grupo de estudo; os animais do grupo CD receberam por gavagem apenas o veículo. As drogas e o placebo foram administrados no período da manhã ao redor das 9 horas, exceto nos animais que receberam ramipril e sinvastatina separadamente (grupo RSS) em que a sinvastatina foi administrada pela manhã, por volta das 9 horas e o ramipril no período da tarde ao redor das 15 horas, nas doses já descritas. Ao final do período de estudo, os animais foram submetidos à anestesia inalatória com éter etílico e receberam injeção intraperitoneal de heparina sódica, diluída $1 / 10 \mathrm{em}$ água destilada estéril, na dose de $500 \mathrm{UI} / \mathrm{kg}$. Após o período de 5 a 8 minutos da administração de heparina, o sangue destes animais foi coletado dos vasos caudais, para determinação de glicemia e do nível de estresse oxidativo. Ao término da coleta, os animais foram mortos por exsanguinação pela seção da aorta abdominal. O número de animais inicialmente alocados para cada grupo foi de oito para os grupos CD, S, R, RSJ e de seis para o grupo RSS. Ocorreram seis óbitos antes do término do estudo (dois no grupo $\mathrm{R}$ e um em cada um dos demais), sempre durante o processo de anestesia inalatória, de maneira que 32 ratos, divididos nos grupos já mencionados, restaram para análise estatística. 


\section{Análise estatística}

Os resultados foram expressos como médias e desviospadrão. A análise estatística de variância (ANOVA), seguida do teste de comparações múltiplas das médias, foi empregada para comparar os grupos de estudo mediante as médias das variáveis quantitativas que apresentassem distribuição gaussiana e variâncias homogêneas. Para as variáveis que violaram a suposição de distribuição gaussiana para realização da ANOVA, foram feitas suas transformações logarítmicas. Para a comparação dos dados de TRAP e de MDA pelo uso da heparina foi utilizado o teste de Mann-Whitney; para as variações de pesos e glicemias dos animais estudados foi utilizado o teste de Kruskal-Wallis. As análises foram realizadas no Guided Data Analysis do programa SAS (Statistical Analysis System). Em todos os testes foi adotado o nível de significância de $5 \%$, considerando-se significantes os resultados com valores de p menor que 0,05.

\section{RESULTADOS}

\section{Avaliação do efeito da injeção intraperitoneal de heparina não-fracionada sobre as defesas antioxidantes e o estresse oxidativo de ratos LEW isogênicos normais (sem diabetes químico)}

Os dados referentes ao efeito da heparina não-fracionada intraperitoneal sobre as defesas antioxidantes e o estresse oxidativo de ratos LEW são apresentados na Tabela 1. A administração de heparina ip, na dose utilizada, não promoveu mudança expressiva no TRAP, mas reduziu significantemente $(\mathrm{p}<0,05)$ os níveis de MDA nos animais heparinizados quando comparado aos controles.
Tabela 1. Valores plasmáticos das defesas antioxidantes (TRAP) e do estresse oxidativo (MDA) nos ratos não-heparinizados (controles) e heparinizados.

\begin{tabular}{lcc}
\hline & $\begin{array}{c}\text { Não- } \\
\text { heparinizados }\end{array}$ & Heparinizados \\
\hline TRAP (média \pm DP) & $116,42 \pm 10,75$ & $117,56 \pm 10,15$ \\
MDA (média \pm DP) & $154,38 \pm 131,47$ & $16,77 \pm 11,62 \S$ \\
\hline
\end{tabular}

$\S=\mathrm{p}<0,05$, Mann-Whitney

\section{Análise do diabetes químico pela estreptozotocina e das defesas antioxidantes/estresse oxidativo nos grupos experimentais propriamente ditos}

\section{Pesos e glicemias}

As glicemias e os pesos não foram significantemente diferentes entre os grupos estudados, quer no momento da administração de estreptozotocina (tempo 0 ), no início da administração das drogas (tempo 1) ou ao final do estudo (tempo 2), pelo teste de Kruskal-Wallis. Os dados de pesos e glicemias destes animais durante o estudo são apresentados na Tabela 2 .

\section{TRAP e MDA}

\section{TRAP}

As defesas antioxidantes plasmáticas estimadas pela TRAP foram significativamente maiores no grupo de animais tratados com ramipril isoladamente $(\mathrm{R})$ e significantemente menores nos animais tratados com ramipril e sinvastatina na mesma gavagem (RSJ); $\mathrm{p}=0,006$ para ambos os grupos, ANOVA/teste de comparações múltiplas das médias.

Tabela 2. Valores médios de peso (g) e de glicemia -glic- (mg/dL) por ocasião da administração de estreptozotocina (tempo 0), por ocasião do início da administração das drogas (†empo 1) e ao final do estudo (†empo 2), nos ratos dos diferentes grupos de estudo.

\begin{tabular}{lcccccc}
\hline Grupo & $\begin{array}{c}\text { Peso } \pm \text { DP } \\
\text { (tempo 0) }\end{array}$ & $\begin{array}{c}\text { Peso } \pm \text { DP } \\
\text { (tempo 1) }\end{array}$ & $\begin{array}{c}\text { Peso } \pm \text { DP } \\
\text { (tempo 2) }\end{array}$ & $\begin{array}{c}\text { Glic } \pm \text { DP } \\
\text { (tempo 0) }\end{array}$ & $\begin{array}{l}\text { Glic } \pm \text { DP } \\
\text { (tempo 1) }\end{array}$ & $\begin{array}{l}\text { Glic } \pm \text { DP } \\
\text { (tempo 2) }\end{array}$ \\
\hline CD & $355,42 \pm 29,34$ & $305,28 \pm 29,91$ & $299,14 \pm 27,69$ & $92,71 \pm 3,30$ & $371,14 \pm 100,50$ & $425,71 \pm 54,29$ \\
\hline R & $365,83 \pm 35,45$ & $311,83 \pm 28,44$ & $284,50 \pm 26,15$ & $90,33 \pm 4,41$ & $341,33 \pm 9,48$ & $460,16 \pm 121,50$ \\
\hline S & $362,42 \pm 45,50$ & $317,28 \pm 27,09$ & $297,42 \pm 30,42$ & $89,42 \pm 2,99$ & $396,21 \pm 105,37$ & $423,28 \pm 104,11$ \\
\hline RSJ & $354,66 \pm 30,94$ & $307,83 \pm 26,27$ & $285,66 \pm 31,65$ & $89,33 \pm 6,18$ & $341,66 \pm 25,40$ & $357,16 \pm 39,15$ \\
RSS & $342,00 \pm 12,88$ & $309,00 \pm 11,81$ & $294,60 \pm 10,31$ & $91,80 \pm 3,19$ & $394,00 \pm 61,37$ & $400,4 \pm 50,16$ \\
\hline
\end{tabular}

$\mathrm{CD}=$ controles diabéticos; $\mathrm{R}$ = diabéticos tratados com ramipril; $\mathrm{S}$ = diabéticos tratados com sinvastatina; RSJ = diabéticos tratados com ramipril e sinvastatina juntos na mesma administração; RSS = diabéticos tratados com ramipril e sinvastatina em administrações separadas. 


\section{$M D A$}

O estresse oxidativo, como medido pela dosagem plasmática de MDA, não foi significantemente diferente entre os grupos (ANOVA, com transformação logarítmica prévia das médias, $\mathrm{p}=0,2148)$.

Os resultados (médias \pm DP) de TRAP e MDA para os diferentes grupos de tratamento são apresentados na Tabela 3.

Tabela 3. Medidas da capacidade antioxidante total plasmática (TRAP) e dos níveis plasmáticos de malonaldeído (MDA) após 2 semanas de tratamento, nos diferentes grupos experimentais.

\begin{tabular}{lcc}
\hline Grupo & TRAP \pm DP & MDA \pm DP \\
CD & $123,09 \pm 16,18$ & $18,73 \pm 16,07$ \\
\hline R & $134,45 \pm 17,94 \S$ & $22,73 \pm 27,28$ \\
S & $109,89 \pm 12,87$ & $26,97 \pm 24,36$ \\
RSS & $110,07 \pm 12,38$ & $4,33 \pm 1,68$ \\
RSJ & $97,01 \pm 5,55 \S$ & $11,64 \pm 13,03$ \\
\hline
\end{tabular}

$C D=$ controles diabéticos; $R$ = diabéticos tratados com ramipril; $S$ = diabéticos tratados com sinvastatina; RSJ = diabéticos tratados com ramipril e sinvastatina juntos na mesma administração; $R S S=$ diabéticos tratados com ramipril e sinvastatina em administrações separadas; $\S=p=0,006$, ANOVA/teste de comparações múltiplas das médias.

\section{DISCUSSÃO}

Para a indução do diabetes experimental foi utilizada a estreptozotocina por via intraperitoneal em vez da via intravenosa, por ser mais prática e conveniente, porque se após duas semanas da indução do diabetes instalado o animal permanecer diabético não mais ocorre reversão espontânea à normoglicemia (17). Em vez da dose usual de $60 \mathrm{mg} / \mathrm{kg}$ de estreptozotocina, como inicialmente pretendido, utilizou-se a dose de $50 \mathrm{mg} / \mathrm{kg}$. Esta escolha deveu-se ao fato de, em estudos preliminares, ter-se observado mortalidade de $53 \%$ com a dose de $60 \mathrm{mg}$, talvez em virtude da idade relativamente avançada dos animais nos experimentos deste estudo comparada à dos animais utilizados no estudo original. Com a dose de $50 \mathrm{mg} / \mathrm{kg}$ nos animais deste trabalho, eliminou-se a grande mortalidade verificada com a dose de $60 \mathrm{mg} / \mathrm{kg}$, alcançando a definição operacional definida para este estudo em todos os animais em que a droga foi administrada. Ressalta-se que, por causa da idade relativamente avançada dos animais estudados, o estresse oxidativo verificado foi a somatória do proveniente do diabetes químico induzido pela estreptozotocina e o do envelhecimento.
Optou-se pela coleta do sangue dos vasos caudais, em vez de punção cardíaca ou por outro método, por ser tecnicamente mais simples e mais prática. Para a heparinização dos animais, padronizou-se $500 \mathrm{UI} / \mathrm{kg}$ após se testar as concentrações de 100, 200, 300, 400 e $500 \mathrm{UI} / \mathrm{kg}$. A concentração de $500 \mathrm{UI} / \mathrm{kg}$ foi a menor concentração testada que produziu o resultado desejado, ou seja, foi a capaz de permitir a coleta de sangue das caudas dos animais, sem que se tivesse de "ordenhá-las" para obter sangue em quantidade suficiente para as determinações bioquímicas, o que aumentava o tempo de coleta e acarretava hemólise.

Sabe-se que a heparina tem início de ação imediata quando administrada por via endovenosa (18); para agilização do estudo, optou-se pela administração intraperitoneal da droga e aguardou-se o tempo de 5 a 8 minutos para que a droga fosse absorvida. A heparina não-fracionada (forma utilizada no estudo) é constituída por um conjunto de macromoléculas com tamanho variável entre 3.000 e $30.000 \mathrm{D}$, que, em sua maioria, teoricamente não cruzariam a membrana peritoneal $(19,20)$. Conseguiu-se heparinização sistêmica apenas pela ação de massa, administrando doses cerca de 5 vezes maiores do que as doses endovenosas necessárias para promover heparinização sistêmica em animais, e acredita-se que a heparinização deu-se mais pela ação das frações menores de heparina, que podem ter atravessado a membrana peritoneal com menor dificuldade. Levou-se em consideração que existem evidências de que a heparina possa exercer atividade antioxidante por reduzir a oxidação da glutationa e por atividade antimieloperoxidase (21). De fato, nos estudos em análise, a heparinização reduziu os níveis de estresse oxidativo significativamente. No entanto, não se pode diferenciar se esta ação foi em razão da heparina ou em virtude da redução no trauma das caudas dos animais pela ordenha para a coleta do sangue, pela redução da hemólise ou pela redução no tempo de coleta. Por estas razões, padronizou-se a dose de heparina e o tempo de coleta do sangue pós-heparina para todos os animais dos diferentes grupos experimentais.

O fato de o procedimento anestésico ter sido aplicado de forma semelhante em todos os animais estudados, até o alcance de anestesia profunda, deve ter controlado para o fato de o anestésico inalatório utilizado (éter etílico) poder aumentar a peroxidação lipídica e, conseqüentemente, os níveis de TBARS (22).

Com o objetivo de se obter a maior penetração celular possível das drogas utilizadas, foram escolhidos 
a sinvastatina e o ramipril, por serem, ambos, os representantes de maior lipossolubilidade entre seus grupos farmacológicos disponíveis no Brasil (23). As doses de $3 \mathrm{mg} / \mathrm{kg}$ de ramipril e de $2 \mathrm{mg} / \mathrm{kg}$ de sinvastatina foram padronizadas neste estudo por já terem sido utilizadas em modelos experimentais semelhantes sobre os efeitos pleiotrópicos de ambas as drogas $(24,25)$. De acordo com Sironi e cols. (26), a dose média efetiva (ED-50) de sinvastatina no rato é de 1,2 $\mathrm{mg} / \mathrm{kg}$; mesmo utilizando dose maior não se verificou atividade antioxidante para a droga. $\mathrm{Na}$ dose de $\mathrm{l}$ $\mathrm{mg} / \mathrm{kg}$ para o rato, o ramipril apresenta efeito antihipertensivo considerado alto (27). Na dose utilizada, a sinvastatina não produz efeito sobre a colesterolemia de ratos (28).

A diluição das drogas foi feita em solução de CMC $0,5 \%$ em vez de água, porque em água as drogas formavam grumos e sua solubilização era difícil; em solução de CMC 0,5\%, a homogeneidade da suspensão para a administração tornava-se melhor.

Apesar de não se ter realizado aferições de pressão arterial nos animais estudados, variável que sabidamente pode interferir no estresse oxidativo por aumentar o cisalhamento do endotélio e, secundariamente, o estresse oxidativo endotelial (29), não se acredita que esta possa ter sido variável de grande importância, pois as defesas antioxidantes, avaliadas pelos níveis plasmáticos de TRAP, aumentaram apenas nos animais que utilizaram ramipril isoladamente, mas não nos animais que utilizaram ramipril e sinvastatina em combinação, e é sabido que o uso de estatinas tende a acarretar discreta redução nos níveis tensionais (12).

Paradoxalmente, a utilização de ambas as drogas em uma única tomada diária resultou piora significativa da capacidade de defesa antioxidante plasmática, o que não ocorreu por ocasião da administração diária de ambas as drogas em gavagens separadas. Considerando-se que neste estudo foram utilizadas as drogas em apresentações comerciais, e que quando elas foram administradas concomitantemente ocorreu redução significativa na defesa antioxidante do plasma, suspeitou-se da possibilidade de haver ocorrido antagonismo farmacodinâmico ou farmacocinético entre as drogas e/ou seus excipientes. Na literatura, é relatado não existir interação medicamentosa entre ramipril e sinvastatina em humanos (30). Deve ser ressaltado que no estudo de Meyer e cols. (30) as drogas foram administradas separadamente, sendo a sinvastatina administrada à noite $\mathrm{e}$ o ramipril pela manhã.
Especula-se que os animais tratados com ramipril isoladamente possam ter tido melhora nos níveis de TRAP por aumento das defesas antioxidantes endoteliais e de células sangüíneas (hemácias especialmente), uma vez que os inibidores de enzima de conversão foram mostrados por aumentarem os níveis celulares de glutationa reduzida, superóxido dismutase e catalase, em modelos experimentais (31-33). Em adição, recentemente Hornig e cols. (34) demonstraram que quatro semanas de uso de ramipril ( $10 \mathrm{mg} /$ dia $)$ ou de losartana - bloqueador dos receptores $\mathrm{AT}_{1}$ da angio II ( $100 \mathrm{mg} /$ dia) resultou mais de $200 \%$ de aumento na atividade da superóxido dismutase extracelular, o principal sistema antioxidante da parede arterial de humanos, sugerindo que tal efeito seja mediado pelo bloqueio das ações da angio II sobre seus receptores $\mathrm{AT}_{1}$. Como o plasma encontra-se em íntimo contato com o endotélio e as hemácias, aumentos nas defesas antioxidantes destes elementos são esperados acarretarem aumentos da TRAP (35). De fato, sabe-se que as hemácias são as principais fontes de defesa antioxidante do sangue e que a anemia está associada ao aumento de estresse oxidativo (36).

Embora a maioria dos estudos indique a ação benéfica das estatinas sobre o estresse oxidativo, estudos recentes têm sugerido que talvez este efeito não seja universal e que dependa do modelo estudado. Neste sentido, a cerivastatina não foi capaz de reduzir o estresse oxidativo de células endoteliais humanas em cultura (37) e o uso clínico de sinvastatina esteve associado a níveis plasmáticos e urinários elevados de isoprostano 8-epi- $\mathrm{PGF}_{2 \alpha}$, marcador de estresse oxidativo, reversíveis com a remoção da droga, em pacientes com dores musculares decorrentes da medicação, mesmo na ausência de elevação dos níveis de creatinofosfoquinase (CPK) (38).

Sabe-se que as estatinas reduzem os níveis de ubiquinona (coenzima Q10 - CQ10), um antioxidante natural das mitocôndrias e membranas lipídicas, conhecido por ser o único antioxidante lipossolúvel sintetizado endogenamente e que tem demonstrado, entre outras atividades, ação preventiva sobre a peroxidação lipídica (39). A redução nos níveis de CQ10 pelas estatinas pode resultar piora do estresse oxidativo em modelos experimentais e em seres humanos (38-42). Utilizando a mesma estimativa das defesas antioxidantes do plasma que a usada no presente estudo (TRAP), Altekin e cols. (43) demonstraram que os níveis da TRAP, bem como os de CQ10, foram reduzidos após 
dois meses de terapia com sinvastatina $(20 \mathrm{mg} / \mathrm{dia}) \mathrm{em}$ pacientes hipercolesterolêmicos.

O fato de nem o ramipril nem a sinvastatina, isoladamente ou combinados, terem sido capazes de reduzir significativamente a peroxidação lipídica medida pelos níveis plasmáticos de MDA foi inesperado, uma vez que estudos prévios demonstraram que a associação destas drogas pode reduzir o estresse oxidativo em estudos experimentais e ensaios clínicos $(14,44,45)$. Os resultados deste estudo devem ser interpretados com cautela, no entanto, uma vez que a heparina administrada aos animais causou profunda redução nos níveis de MDA plasmáticos (redução no estresse oxidativo), não permitindo, talvez, a observação de possível ação benéfica destas drogas sobre a peroxidação lipídica.

Em conclusão, o ramipril, usado isoladamente, aumentou significativamente as defesas antioxidantes plasmáticas do rato diabético, como medidas pelos níveis de TRAP plasmáticos, enquanto a sinvastatina, dada isoladamente ou combinada ao ramipril em tomadas separadas, não produziu efeito significativo sobre estas defesas antioxidantes. Paradoxalmente, a administração concomitante das duas drogas reduziu significativamente as defesas antioxidantes destes animais, talvez por interação farmacocinética/farmacodinâmica. Nenhuma das drogas utilizadas foi capaz de reduzir o nível de estresse oxidativo estimado pela determinação plasmática de MDA, sendo uma possibilidade para a ausência deste efeito o uso de heparina nos animais estudados. Estudos experimentais futuros que venham avaliar a ação antioxidante destas drogas, a partir da análise dos níveis séricos de MDA, devem evitar a heparinização dos animais de estudo para suprimir este possível viés da análise. Os dados do presente trabalho corroboram o efeito positivo do ramipril sobre a defesa antioxidante do plasma, mas não confirmam possível efeito benéfico da sinvastatina, no modelo estudado. Pesquisas adicionais são necessárias para definir se a redução das defesas antioxidantes plasmáticas dos ratos com diabetes experimental foi por causa da interação farmacodinâmica/ farmacocinética das apresentações farmacêuticas utilizadas ou secundária a outro(s) fator(es), definir melhor o papel destas drogas sobre o estresse oxidativo do diabetes melito experimental e clínico e estabelecer qual a relevância que o possível efeito benéfico destas drogas sobre o estresse oxidativo representaria, em adição ao controle da hipertensão e da hipercolesterolemia, na redução da morbimortalidade de indivíduos diabéticos.
Agradecimentos. Agradecemos à Professora Maria Emilia Fávero, responsável pelo Laboratório de Pós-graduação do Centro de Ciências da Saúde da UEL, pelo auxílio na execução do projeto. Conflito de interesses: Trabalho realizado no Laboratório de Pósgraduação do Hospital Universitário Norte do Paraná, Centro de Ciências da Saúde da UEL, como parte de dissertação de mestrado em Medicina Interna e Ciências da Saúde da UEL de José Antonio Zarate Elias. Os autores declaram não haver conflito de interesse. Os autores declaram não haver conflitos de interesse científico neste artigo.

\section{REFERÊNCIAS}

1. Delfino VDA, Mocelin AJ. Transplante de pâncreas e ilhotas pancreáticas: visão de nefrologista. Arq Bras Endocrinol Metab. 2002;46(2):177-85.

2. Kimura F, Hasegawa G, Obayashi $H$, Adachi T, Hara H, Ohta $M$, et al. Serum extracellular superoxide dismutase in patients with type 2 diabetes. Diabetes Care. 2003;26:1246-50.

3. Miwa MS, Inouye C, Ohnura C, Mitsuhashi N, Onuma T, Kawamori R. Relationship between carotid atherosclerosis and erythrocyte membrane cholesterol oxidation products in type 2 diabetic patients. Diabetes Res Clin Pract. 2003;61:81-8.

4. Nascimento NRF, Costa-E-Forti A, Peter AA, Fonteles MC. Free radical scavengers improve the impaired endotheliumdependent responses in aorta and kidneys of diabetic rabbits. Diabetes Res Clin Pract. 2003;61:145-53.

5. Faria AN, Zanella MT, Kohlman O, Ribeiro AB. Tratamento de diabetes e hipertensão no paciente obeso. Arq Bras Endocrinol Metab. 2002;46(2):137-42.

6. Johansen JS, Harris AK, Rychly DJ, Ergul A. Oxidative stress and the use of antioxidants in diabetes: linking basic science to clinical pratice. Cardiovasc Diabetol. 2005;4:5. Review.

7. Münzel T, Keaney Jr JF. Are ACE inhibitors a "magic bullet" against oxidative stress? Circulation. 2001;104:1571-4.

8. Davignon J, Jacob RF, Mason RP. The antioxidant effect of statins. Coron Artery Dis. 2004;15:251-8.

9. Schulz I. Treatment of dyslipidemia: how and when to combine lipid lowering drugs. Arq Bras Endocrinol Metab. 2006 50(2):344-59.

10. Ceriello A. New insights in oxidative stress and diabetic complications may lead to a "causal" antioxidant therapy. Diabetes Care. 2003;26(5):1589-96.

11. Kuroki T, Isshiki K, King GL. Oxidative stress: the lead or supporting actor in the pathogenesis of diabetic complications. $J$ Am Soc Nephrol. 2003;14(Suppl 3):216-20.

12. Mcfarlane SI, Muniyappa R, Francisco R, Sowers JR. Pleiotropic effects of statins: Lipid reduction and beyond. J Clin Endocrinol Metabol. 2002;87(4):1451-8.

13. Melhem MF, Craven PA, Derubertis FR. Effects of supplementation of $\propto$-lipoic acid on early glomerular injury in diabetes mellitus. J Am Soc Nephrol. 2001;12:124-33.

14. Onozato ML, Tojo A, Goto A, Fujita T, Wilcox CS. Oxidative stress and nitric oxide synthase in rat diabetic nephropathy: Effects of ACEI and ARB. Kidney Int. 2002;61:186-94.

15. Position Statement of the American Diabetes Association Responsible use of animals in research. Diabetes Care. 1990;13(Suppl 1):38. 
16. Barbosa DS, Cecchini R, El Kadri MZ, Rodriguez MAM, Burini $\mathrm{RC}$, Dichi I. Decreased oxidative stress in patients with ulcerative colitis supplemented with fish oil w-3 fatty acids. Nutrition. 2003;19:837-42.

17. Delfino VDA, Figueredo J, Matsuo T, Favero ME, Matni AM, Mocelin AJ. Diabetes mellitus induzido por estreptozocina: comparação em longo prazo entre duas vias de administração. J Bras Nefrol. 2002;24(1):31-6.

18. Hirsh J, Warkentin TE, Shaughnessy SG, Anand SS, Halperin $\mathrm{JL}$, Raschke R, et al. Heparin and low-molecular-weight heparin mechanisms of action, pharmacokinetics, dosing, monitoring, efficacy, and safety. Chest. 2001;119(Suppl ):64S-94S.

19. Kaplan GG, Manns B, Mclaughlin K. Heparin induced thrombocytopaenia secondary to intraperitoneal heparin exposure. Nephrol Dial Transplant. 2005;20:2561-2.

20. Takahashi S, Shimada A, Okada K, Kuno T, Nagura Y, Hatano $M$. Effect of intraperitoneal administration of heparin to patients on continuous ambulatory peritoneal dialysis (CAPD). Perit Dial Int. 1991;11:81-3.

21. Sela S, Shurtz-Swirski R, Shapiro G, Nasser L, Hamzi M, Shasha SM, et al. Oxidative stress during hemodialysis: effect of heparin. Kidney Int. 2001;59(Suppl 78):159-63.

22. Liu PT, Kentish PA, Symons AM, Parke DV. The effects of ether anaesthesia on oxidative stress in rats-dose response. Toxicology. 2003;4,80(1):37-49.

23. Tomlinson B, Chan P, Lan W. How well tolerated are lipid-lowering drugs? Drugs Aging. 2001;18(9):665-83.

24. Li C, Yang CW, Park CW, Ahn HJ, Kim WY, Yoon KH, et al. Longterm treatment with ramipril attenuates renal osteopontin expression in diabetic rats. Kidney Int. 2003;63:454-63.

25. Oin J, Zhang Z, Liu J, Sun L, Hu L, Cooper ME, et al. Effects of the combination of an angiotensin II antagonist with an HMGCoA redutase inhibitor in experimental diabetes. Kidney Int. 2003;61:565-71.

26. Sironi L, Gianazza E, Gelosa P, Guerrini U, Nobili E, Gianella $A$, et al. Rosuvastatin, but not simvastatin, provides end-organ protection in stroke-prone rats by antiinflammatory effects. Arterioscler Thromb Vasc Biol. 2005;25:598-603.

27. Linz W, Jessen T, Becker RHA, Schölkens BA, Wiemer G. Long-term ACE inhibition doubles lifespan of hypertensive rats. Circulation. 1997;96:3164-72.

28. Zoja C, Corna D, Rotolli D, Cattaneo D, Zanchi C, Tomasoni S, et al. Effect of combining ACE inhibitor and statin in severe experimental nephropathy. Kidney Int. 2002;61:1635-45.

29. Mehta JL, Chen J, Hermonat PL, Romeo F, Novelli G. Lectinlike, oxidized low-density lipoprotein receptor-1 (LOX-1): a critical player in the development of atherosclerosis and related disorders. Cardiovasc Res. 2006;69:36-45.

30. Meyer BH, Scholtz HE, Muller FO, Luus HG, De La Rey N, Seibert-Grafe M, et al. Lack of interaction between ramipril and simvastatin. Eur J Clin Pharmacol. 1994;47:373-5.

31. Anderson B, Khaper N, Dhalla AK, Singal PK. Anti-free radical mechanisms in captopril protection against reperfusion injury in isolated rat hearts. Can J Cardiol. 1996;12(10):1099-104.

32. de Cavanagh EM, Inserra F, Ferder LN, Fraga CG. Enalapril and captopril enhance glutathione-dependent antioxidant defenses in mouse tissues. Am J Physiol Regul Integr Comp Physiol. 2000;278:572-7.

33. Sugimoto K, Tsuruoka S, Fujimura A. Effect of enalapril on diabetic nephropathy in oletf rats: the role of an anti-oxidative action in its protective properties. Clin Exp Pharmacol Physiol. 2001;28:826-30.

34. Hornig B, Landmesser U, Kohler C, Ahlersmann D, Spiekermann S, Christoph A, et al. Comparative effect of ACE inhibition and angiotensin II typo 1 receptor antagonism on bioavailability of nitric oxide in patients with coronary artery disease, role of superoxide dismutase. Circulation. 2001;103: 799-805.

35. Torres RL, Torres ILS, Gamaro GD, Fontella FU, Silveira PP, Moreira JSR, et al. Lipid peroxidation and total radical-trapping potential of the lungs of rats submitted to chronic and sub-chronic stress. Braz J Biol Res. 2004;37:185-92.

36. Grune T, Sommerburg O, Siems Wg. Oxidative stress in anemia. Clin Nephrol. 2000;53(Suppl 1):18-22.

37. Al-Ruzzeh S, Schimidt I, Nakamura K, Chester A, lisley C, Anrani $M$. Cerivastatin does not prevent oxidative injury of human aortic endothelial cells. Tex Heart Inst J. 2004;31:127-31.

38. Sinzinger $\mathrm{H}$, Lupattelli $\mathrm{G}$, Oguogho A, Furberg $C D$. Isoprostane 8 -epi-PGF2 $\alpha$ is frequently increased in patients with muscle pain and/or CK-elevation after HMG-Co-enzyme-A-reductase inhibitor therapy. J Clin Pharm Ther. 2001;26:303-10.

39. Bonakdar RA, Guarnieri E. Coenzyme Q10. Am Fam Physician. 2005;72:1065-70.

40. Beltowski J. Statins and modulation of oxidative stress. Toxicology Mech Meth. 2005;15(2):61-92.

41. Cenedella RJ, Neely AR, Sexton P. Concentration and distribution of ubiquinone (coenzyme $Q$ ), the endogenous lipid antioxidant, in the rat lens: effect of treatment with simvastatin. Mol Vis. 2005;11:594-602.

42. Passi S, Stancato A, Aloe E, Dmitrieva A, Littarru GP. Statins lower plasma and lymphocyte ubiquinol/ubiquinone without affecting other antioxidants and PUFA. Biofactors. 2003;18/14):113-24.

43. Altekin E, Onvural B, Çoker C, Guneri S, Çaliskan S. The effect of simvastatin treatment on plasma ubiquinone, blood ATP concentrations, total antioxidant capacity and muscle related markers. J Trace Elem Med Biol. 2002;32:323-8.

44. Koh KK, Son JW, Ahn JY, Kim DS, Jin DK, Kim HS, et al. Simvastatin combined with ramipril treatment in hypercholesterolemic patients. Hypertension. 2004;44:180-5.

45. Koh KK, Quon MJ, Han SH, Ahn JY, Jin DK, Kim HS, et al. Vascular and metabolic effects of combined therapy with ramipril and simvastatin in patients with type 2 diabetes. Hypertension. 2005;45:1088-93.

\section{Endereço para correspondência:}

José Antonio Zarate Elias

Av. Paris, 675 - Jardim Piza

86041-100 Londrina, PR

E-mail: jazelias@yahoo.com.br 